\title{
Imaging Unsteady Propagation of Reaction Fronts in Reactive Multilayer Foils with Multi-Frame Dynamic TEM
}

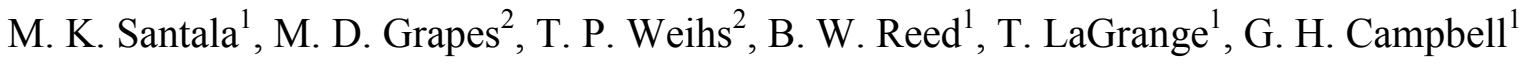 \\ 1. Condensed Matter \& Materials Division, Lawrence Livermore National Laboratory, Livermore, USA \\ 2. Department of Materials Science and Engineering, The Johns Hopkins University, Baltimore, USA
}

Reactive multilayer foils (RMLFs) are composites formed of thin alternating layers of metals that undergo exothermic mixing reactions to form intermetallic compounds. The exothermic reaction provides adequate energy to cause self-propagation of the reaction beyond the local area where the reaction is initiated by an external energy source. RMLFs are used as local heat sources for brazing and soldering as well as for other commercial and defense applications.

The energy release and the rate of the reaction propagation may be regulated through the chemistry and bilayer thickness of the multilayer foils. Reaction velocities have been characterized using photodiode arrays [1] and high-speed cameras [2]. Single-shot dynamic transmission electron microscopy (DTEM) has also been used to study transient structures in the reaction front of Al-Ni multilayer foils and characterize the reaction rate during steady propagation $[3,4]$.

In this work, we use multi-frame DTEM movies to image the self-propagating reaction in aluminumrich $\mathrm{Ni}$-Al nanolaminate RMLFs which have been designed to undergo unsteady reaction propagation after local initiation with a pulsed laser. During unsteady propagation, radial growth of the reacted area occurs by the successive reaction of narrow bands of material in which the reaction front propagates tangentially along recently reacted material. Unsteady, or "spinlike", propagation had been observed in other RMLF systems, eg. [5,6] and has been modeled in 3-D numerical simulations [7].

Preliminary DTEM results for specimens with eight $25 \mathrm{~nm}$-thick bilayers and an overall composition of $\mathrm{Al}_{.72} \mathrm{Ni}_{28}$ show the reaction front is initially steady and propagates at speeds in excess of $10 \mathrm{~m} / \mathrm{s}$. As the reaction front grows outward from the initiation point toward cooler parts of the foil the reaction front slows and propagation becomes unsteady (Figure $1 \mathrm{a}-\mathrm{f}$ ). Results from the DTEM experiments will be compared to recent models of unsteady RMLF propagation $[7,8]$.

\section{References:}

[1] M. E. Reiss et al., Materials Science and Engineering A 261 (1999), p. 217.

[2] L. A. Clevenger et al., Journal of Applied Physics 67 (1990), p. 2894.

[3] J. S. Kim et al., Science 321 (2008), p. 1472.

[4] J. S. Kim et al., Acta Materialia 59 (2011), p. 3571.

[5] J. C. Trenkle et al., Applied Physics Letters 87 (2005), p. 153108.

[6] J. P. McDonald et al., Applied Physics Letters 94 (2009), p. 34102.

[7] L. Alawieh et al., Journal of Applied Physics 110 (2011), p. 13509.

[8] This work performed under the auspices of the U.S. Department of Energy, Office of Basic Energy Sciences, Division of Materials Sciences and Engineering by Lawrence Livermore National Laboratory under Contract DE-AC52-07NA27344. 

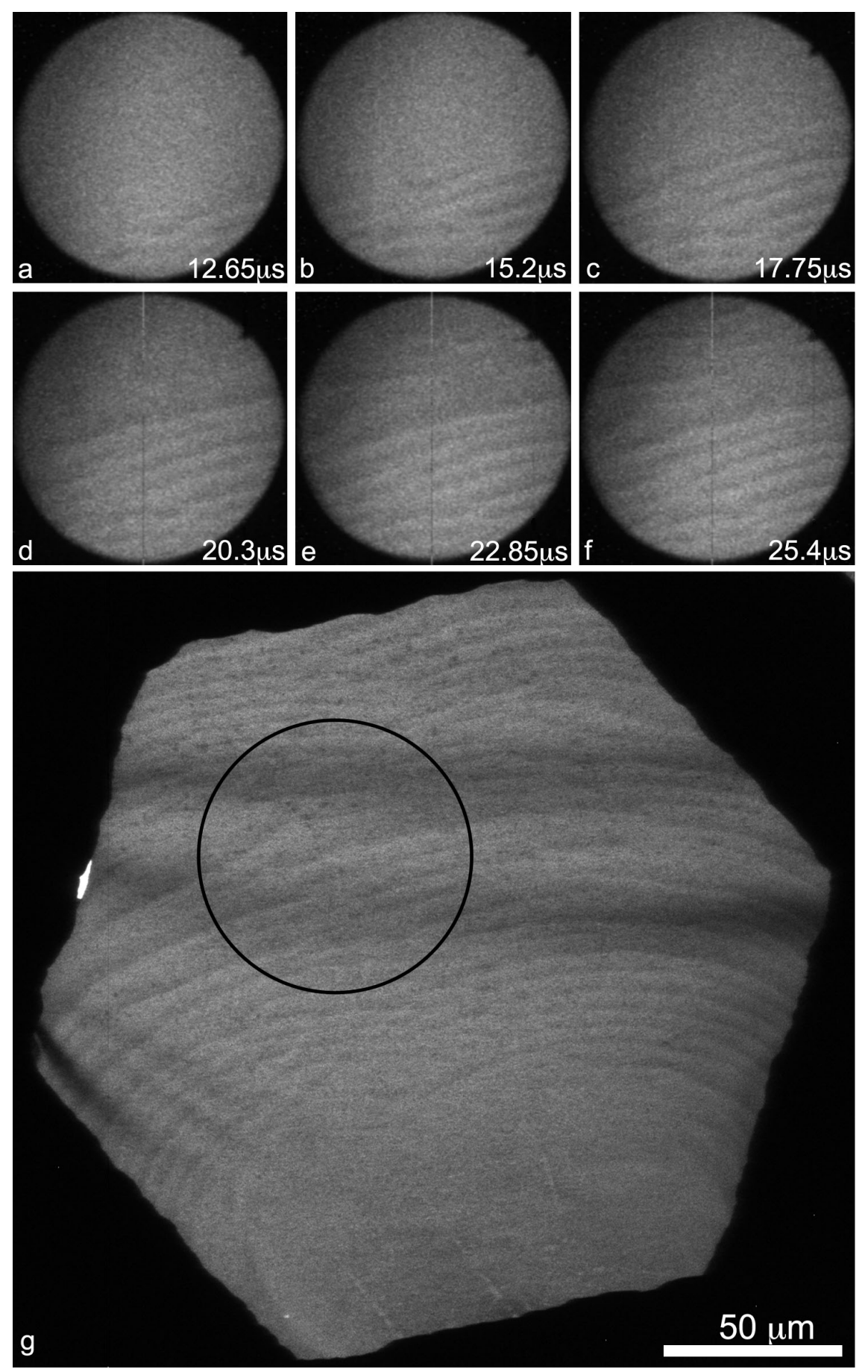

Figure 1. (a-f) Pulsed electron images showing the unsteady propagation of the self-sustaining part of the mixing reaction. Each frame in (a-f) is formed with a 50-ns electron pulse. The time signature in each frame is relative to the time of the peak specimen laser intensity with an uncertainty of $\pm 3 \mathrm{~ns}$. (g) A conventional bright field TEM image minutes after the reaction. The circle in (g) indicates the region imaged in the time resolved images in (a-f). The laser pulse initiating the reaction was centered at the bottom 